\title{
Longitudinal investigation of the role of temperament and stressful life events in childhood anxiety
}

Article

Accepted Version

Broeren, S., Newall, C., Dodd, H. and Hudson, J. L. (2014) Longitudinal investigation of the role of temperament and stressful life events in childhood anxiety. Development and Psychopathology, 26 (2). pp. 437-449. ISSN 1469-2198 doi: https://doi.org/10.1017/S0954579413000989 Available at https://centaur.reading.ac.uk/33951/

It is advisable to refer to the publisher's version if you intend to cite from the work. See Guidance on citing.

To link to this article DOI: http://dx.doi.org/10.1017/S0954579413000989

Publisher: Cambridge University Press

Publisher statement: This is the author's copy of the manuscript. The paper has been accepted for publication and will appear in a revised form, subsequent to editorial input by Cambridge University Press in Development and Psychopathology, published by Cambridge University Press. Copyright Cambridge University Press. http://journals. cambridge.org/action/displayJournal?jid=DPP

All outputs in CentAUR are protected by Intellectual Property Rights law, including copyright law. Copyright and IPR is retained by the creators or other copyright holders. Terms and conditions for use of this material are defined in the End User Agreement. 


\section{www.reading.ac.uk/centaur}

\section{CentAUR}

Central Archive at the University of Reading

Reading's research outputs online 
Broeren, S., Newall, C., Dodd, H.F., \& Hudson, J.L. (accepted May 2013). Longitudinal investigation of the role of temperament and stressful life events in childhood anxiety. Development and Psychopathology.

This is the author's copy of the manuscript. The paper has been accepted for publication and will appear in a revised form, subsequent to editorial input by Cambridge University Press in Development and Psychopathology, published by Cambridge University Press. Copyright Cambridge University Press. http://journals.cambridge.org/action/displayJournal?jid=DPP 


\begin{abstract}
The current study investigated the longitudinal relationships between BI, life events, and anxiety in a sample of 102 behaviourally inhibited (BI) and 100 uninhibited (BUI) children aged 3 to 4 years. Children's parents completed questionnaires on BI, stressful life events, and anxiety symptoms, and were administered a diagnostic interview three times in a 5-year period. In line with our hypotheses, negative life events, and negative behaviourdependent life events (i.e. life events that are related to the children's own behaviours) in particular, and the impact of negative life events, were predictive of increases in subsequent anxiety symptoms, the likelihood of having an anxiety disorder, and increased number of anxiety diagnoses over the five year follow-up period. Experiencing more positive, behaviour-independent life events decreased the risk of being diagnosed with an anxiety disorder. Furthermore, differences were found in life events between BI and BUI children. That is, BI children experienced fewer positive and specifically positive behaviour-dependent life events, and the impact of these positive life events was also lower in BI children than in BUI children. However, BI did not interact with life events in the prediction of anxiety problems as hypothesized. Therefore, this study seems to indicate that BI and life events act as additive risk factors in the development of anxiety problems.
\end{abstract}

Keywords: Anxiety disorders; Children, Life events; Behavioural inhibition. 
RUNNING HEAD: TEMPERAMENT, STRESSFUL LIFE EVENTS, AND ANXIETY

Longitudinal investigation of the role of temperament and stressful life events in childhood anxiety

Behavioural inhibition (BI) is a temperament trait that is characterised by the tendency to be unusually shy and to respond to unfamiliar social and non-social situations with extreme apprehension, fearfulness, and withdrawal (Hirshfeld-Becker, Biederman, \& Rosenbaum, 2004). Approximately 15 percent of children exhibit this trait (Kagan, Reznick, Clarke, Snidman, \& Garcia-Coll, 1984), which has a genetic basis (Robinson, Kagan, Reznick, \& Corley, 1992) and shows moderate to considerable stability over childhood (e.g. Biederman et al., 1993; Hirshfeld et al., 1992; Kerr, Lambert, Stattin, \& KlackenbergLarsson, 1994; Prior, Smart, Sanson, \& Oberklaid, 2000; Reznick, Gibbons, Johnson, \& McDonough, 1989; Scarpa, Raine, Venables, \& Mednick, 1995). Research shows that BI children, and in particular the ones that show stable high BI during childhood (e.g. Hirshfeld et al., 1992), are at heightened risk for developing an anxiety disorder in general, and especially social phobia (e.g. Biederman et al., 1993; Hudson, Dodd, Lyneham, \& Bovopoulous, 2011; Muris, Van Brakel, Arntz, \& Schouten, 2011).

However, despite the established link between BI and the development of childhood anxiety disorders, many BI children do not go on to develop an anxiety disorder later in life (Biederman et al., 1993; Prior et al., 2000). Moreover, not all individuals suffering from anxiety disorders were BI as children (Turner, Beidel, \& Wolff, 1996). This raises the question under what conditions BI children develop anxiety disorders. Various authors have proposed that BI serves as a vulnerability factor for anxiety within a diathesis-stress model (Biederman, Rosenbaum, Chaloff, \& Kagan, 1995; Muris, Merckelbach, Schmidt, Gadet, \& Bogie, 2001; Turner et al., 1996). This model presumes that, due to an endogenous vulnerability factor (e.g. BI), some children are more susceptible to the adverse effects of negative experiences and exposures (e.g. stressful life events; Belsky \& Pluess, 2009). That 
is, the temperament of the child interacts with negative experience (i.e., stress) in the onset of psychopathology. In the case of anxiety disorders, Turner and colleagues (1996) suggested that BI children are more likely than behaviourally uninhibited (BUI) children to respond intensely to anxiety-provoking events, and are therefore vulnerable to the development of anxiety problems.

Currently, there is increasing evidence that difficult temperament such as BI or certain gene polymorphisms that have been associated with this temperamental trait (e.g. 5-HTTLPR short alleles) not only make children most adversely affected when confronted with negative environments and stressors, but also make them benefit most from positive environments (Belsky, Bakermans-Kranenburg, \& van IJzendoorn, 2007; Belsky \& Pluess, 2009). For example, while it is unsurprising that children with difficult/fearful temperament show the greatest escalation of problem behaviours in negative environments (i.e., harsh and controlling parenting) compared to children with easy temperaments, difficult/fearful temperament children also show the least increase in problem behaviours when reared in positive environments (i.e., warm and sensitive parenting) (e.g. Bradley \& Corwyn, 2008; Lengua, 2008; Pluess \& Belsky, 2010; Stright, Gallagher, \& Kelley, 2008; van Aken, Junger, Verhoeven, van Aken, \& Deković, 2007). The diathesis-stress model cannot explain these finding as it does not include variation in responses to positive experiences. If anything, this viewpoint suggests that there should be no differences between vulnerable and resilient children in the absence of negative experiences (Pluess and Belsky, 2012). An alternative model has been proposed to explain the findings on positive environment. The model is called the 'differential-susceptibility hypothesis'. This model presumes that some children are more responsive their environment than other children. That is, they stand to benefit most from positive environments but are also most vulnerable to the negative effects of adverse experiences and exposures (Belsky \& Pluess, 2009). Differential susceptibility is, however, 
ideally based on investigating contextual conditions that range from positive to negative. However, some research findings suggest that this assumption also has its limitations. For example, children with a high IQ are highly responsive to supportive environments (Sameroff, 2000) but they are also unresponsive to and protected from aversive environments (e.g. Rutter, 1987). Therefore, Pluess and Belsky (2012) have proposed an additional model called 'vantage-sensitivity' to characterize variability in response to exclusively positive experiences as a function of individual endogenous characteristics. So far, no study has explicitly looked at the interaction between $\mathrm{BI}$ and negative but also positive life events, and anxiety, and it is unknown which of these models best describes the interaction between BI and life events in predicting anxiety problems early in life.

Negative life events have been shown to play a role in the development of childhood anxiety disorders (Allen, Rapee, \& Sandberg, 2008; Boer et al., 2002; Edwards, Rapee, \& Kennedy, 2010; Eley \& Stevenson, 2000; Goodyer, Wright, \& Altham, 1990; Rapee \& Szollos, 2002; Tiet et al., 2001). More specifically, research has revealed that anxious children experience a larger number (Allen et al., 2008; Boer et al., 2002; Eley \& Stevenson, 2000; Goodyer et al., 1990; Rapee \& Szollos, 2002) and greater impact of negative life events (Boer et al., 2002; Gothelf, Aharonovsky, Horesh, Carty, \& Apter, 2004) than non-anxious control children. It has been difficult for researchers to ascertain whether life events play a causal role in the development of anxiety disorders, at least in part because of the chronic nature of anxiety, which makes it difficult to establish the exact onset of an anxiety disorder. Yet, one study has shown that anxious children report a higher number of negative life events in the year preceding anxiety onset compared to non-anxious children, suggesting that life events may affect anxiety over time (Allen et al., 2008). Furthermore, most studies have focused on negative life events only and do not distinguish between life events that partly related to the children's own behaviours (behaviour-dependent events), such as conflicts with 
parents or peers, and those that are independent of children's behaviour (e.g. natural disasters). However, there is some initial evidence that anxious children might experience more behaviour-dependent negative life events and less behaviour-dependent positive life events (Allen \& Rapee, 2009), as well as more behaviour-independent negative life events (Allen et al., 2008; Eley \& Stevenson, 2000). Some researchers have proposed that the distinction between behaviour-dependent and behaviour-independent negative life events might be important because anxious children may behave in a manner that can wholly or partially perpetuate negative life experiences, which in turn can maintain ongoing anxiety problems (Sandberg, McGuinness, Hillary, \& Rutter, 1998). Furthermore, it is essential to also consider variations between individuals in response to positive events, as results of previous studies suggest that positive life events directly influence children's anxiety by decreasing distress and/or indirectly act as a buffer against the impact of negative life events (Garnefski, Kraaij, \& Spinhoven, 2001; Shahar \& Priel, 2002). Initial evidence suggests that anxious children experience less behaviour-dependent positive life events (Allen \& Rapee, 2009), perhaps because they are less likely to initiate positive experiences (e.g. new friendships, participating in team sports and activities) due to their anxiety and associated avoidance.

To date, the influence of life events, and also BI, on anxiety has primarily been assessed in a main-effects model, instead of in a diathesis-stress/vantage sensitivity or differential susceptibility model. Only three studies are available that have assessed the role of BI together with stressful life events in the development of anxiety problems, with mixed results (Brozina \& Abela, 2006; Edwards et al., 2010; Muris et al., 2011). Muris et al (2011) and Edwards et al (2010) showed that BI and negative life events were independent predictors of anxiety symptoms, but found no support for negative life events moderating the relationship between BI and anxiety (i.e., BI interacting with negative environments to lead to 
onset of anxiety). In contrast, Brozina and Abela (2006) found support for such a relationship: Children with high levels of BI showed increased anxiety levels at 6-week follow-up when they experienced high levels of stress caused by negative life events. Differences in results between these studies could have been due to methodological differences (e.g. use of different measures to assess life events) or differences in the length of follow-up. These studies have also focussed on the development of anxiety symptoms, but no studies to date have used diagnostic measures to assess the development of childhood anxiety disorders. Additionally, these studies did not differentiate between behaviour-dependent and behaviour-independent life events and did not include assessment of positive life events.

With these issues in mind, the current study will assess the longitudinal relationship between life events, BI and anxiety in a sample of young children over a 5-year period. The study will not only include a measure of anxiety symptoms as an outcome measure, but will also include a diagnostic interview to check for the presence of actual anxiety disorders. Furthermore, a differentiation will be made between behaviour-dependent and behaviourindependent life events as well as positive and negative life events and the impact of the life events. Based on the literature described above, a number of hypotheses were tested. First, we predict that higher numbers of negative life events and higher impact of negative life events will predict more anxiety at 5-year follow-up, whereas higher numbers of positive life events and higher impact of positive life events will predict less anxiety. Second, consistent with findings in anxious children, BI children will display higher levels of negative, behaviourdependent and behaviour-independent, life events and fewer positive life events than BUI children. Third, consistent with the diathesis-stress and differential susceptibility model, BI will interact with number of negative life events or impact of negative life events to predict anxiety over time. Finally, consistent with the vantage sensitivity and differential susceptibility model, BI will also interact with the number and impact of positive life events 
such that BI children will display lower levels of anxiety over time. No further hypotheses were tested regarding the distinction between behaviour-dependent or independent events in predicting the onset of anxiety problems given the lack of empirical evidence on this issue. However, the role of these different types of life events will also be explored in the current study.

\section{Methods}

This research is part an extensive ongoing longitudinal research project following a sample of behaviourally inhibited (BI) and uninhibited children (BUI) and their parents. A detailed description of the sample and measures from previous data waves (i.e. baseline and 2-year \& 5-year follow-up) can be found in earlier publications (REFERENCES BLINDED FOR REVIEW).

\section{Participants}

Two hundred and two children (102 BI and $100 \mathrm{BUI}$; $M$ age $=48.21$ months, $S D=$ 4.25) participated in this study. They were recruited from local preschools and via an advertisement in a free parenting magazine and participated in the baseline assessment of this study. In total 2182 screening questionnaires were distributed and 567 (26\%) were returned. Children scoring one SD above or below the normative mean score of the Approach subscale of the mother-reported Short Temperament Scale for Children (STSC) (Sanson, Smart, Prior, Oberklaid, \& Pedlow, 1994) were classified as BI or BUI respectively $(\mathrm{N}=317)$.

Subsequently, these children were invited to participate in the full study and $202(64 \%)$ agreed. Children with a developmental disorder or had parents who were unable to read a standard English newspaper were excluded from the study. Eighty-eight percent of these children participated in the 2-year follow-up (87 BI and $91 \mathrm{BUI})$, and 79 percent (71 BI and 89 BUI) participated in the 5-year follow-up. In general, at baseline, most children lived with both parents $(88.6 \%)$ and families had average to above average incomes (respectively $29.1 \%$ 
and $58.7 \%)$. The majority of the sample was of Oceanic ${ }^{1}$ ethnicity $(64.7 \%)$, whereas the remainder of the sample predominantly was from European (18.9\%) or Asian $(10.0 \%)$ ethnicity. Finally, most mothers finished post-school qualifications (84.5\%) and either worked part-time $(42.3 \%)$ or stayed home by choice $(49.3 \%)$. There were no significant differences between families that participated and drop-outs in demographics, except that drop-outs were more likely to have been classified as $\mathrm{BI}, \chi^{2}(1)=11.53, p=.001$, and were more likely to meet the criteria for an anxiety disorder at baseline, $\chi^{2}(1)=7.93, p=.005$.

At baseline, BI and BUI groups did not differ on age, gender, family income and structure, number of siblings, birth order, maternal age or education (for more details about the sample at baseline see REFERENCES BLINDED FOR REVIEW). The only difference between BI and BUI groups was that BI children were more likely of Asian ethnicity, $\chi^{2}(5)=$ $11.871, \mathrm{p}=.04$

\section{Measures}

Behavioural inhibition. At baseline, BI was assessed with the STSC Approach subscale scores as rated by the mother. This STSC Approach subscale contains of 7 items covering the tendency to approach versus withdrawal from novel social and non-social situations. The STSC displays good reliability and adequate validity (Sanson et al., 1994). At baseline, the internal consistency $(\alpha)$ for the STSC Approach scale in the current sample was .92.

Next to mother report, BI was also assessed using a series of laboratory observation tasks (Kagan, Reznick, \& Gibbons, 1989). Children's responses to various novel social (masked experimenter dressed in a strange suit and same-sex peer) and non-social situations (room and toy) were observed. The following behaviour domains were rated: a) time spent

\footnotetext{
${ }^{1}$ Oceanic/Oceania is a new category introduced by the Australian Bureau of Statistics for standard classification of cultural and ethnic groups in 2011. This group includes Australians, Australian Aboriginals, Australian South Sea Isnalder and Torres Strait Islander. All participants who identified as 'Australians' are classified under the category of 'Oceanic'.
} 
proximal to mother, b) amount of time staring at peer, c) time spent talking, d) number of approaches to stranger, and e) number of approaches to peer. A predetermined cut-off (i.e. displaying inhibition in three or more of these five domains) was used to categorize children as BI or BUI based on observation (Rapee, Kennedy, Ingram, Edwards, \& Sweeney, 2005). Inter-rater reliability was determined based on a second trained coder's independent judgment of $25 \%$ of the videotaped sessions. The inter-rater reliability of the number of domains that children displayed inhibited behaviours on (or number of cut-offs exceeded) was ICC $=.91$, and kappa was .79 for the overall BI classification.

Child anxiety. Mothers completed the 32 item Preschool Anxiety Scale, an agedownward adaptation of Spence Children's Anxiety Scale (PAS; Spence, Rapee, McDonald, \& Ingram, 2001) at baseline, and the 40 item Spence Children's Anxiety Scale (SCAS) (Nauta et al., 2004) at 5-year follow-up. In this study, the total scales of these questionnaires are used as a measure of child anxiety symptoms. The PAS and SCAS total scale have satisfactory reliability and validity in preschool and school-aged samples (Broeren \& Muris, 2008; Nauta et al., 2004; Spence et al., 2001). Internal consistency for the PAS and SCAS scales was .93 at baseline and 5-year follow-up.

Additionally, the Anxiety Disorders Interview Schedule for DSM-IV, parent/child version (ADIS-P-IV) (Silverman \& Nelles, 1988) was used to check for the presence of a clinical anxiety disorder and number of anxiety diagnoses at baseline and 5-year follow-up. At baseline, diagnoses were only based on parent-report, whereas at 5-year follow-up both parent and child were interviewed and both responses were used to obtain composite diagnoses. Trained graduate psychologists unaware of the child's BI group membership assigned diagnosis and Clinical Severity Ratings (CSRs). Diagnoses were only considered 'clinical' if the CSR was 4 or greater (on a 0-8 scale). 
To assess inter-rater reliability, a second graduate psychologist coded $20 \%$ of the videotaped interviews. Satisfactory inter-rater reliability was found for presence of a clinical anxiety diagnosis (baseline kappa $=.86,5$-year follow-up kappa $=.85)$ and number of anxiety diagnoses (baseline ICC $=.90,5$-year follow-up ICC $=.90)$.

Stressful life events. At 2-year follow-up mothers completed the Child and Adolescent Survey of Experiences (CASE-P) (Allen et al., 2012). In this measure, mothers indicate whether each of 38 life events occurred to their child in the 12 month period prior to completion. When a life event had occurred, mothers were asked to provide a rating for how good or bad the event was for their child (impact) using a 6-point scale from Really good to Really bad. This rating was used to categorize events as positive or negative (on an individual basis). Furthermore, the distinction between dependent and independent life events was based on the categorisation that Allen and Rapee (2009) made based on consensus judgement by the authors and past research (see Allen and Rapee, 2009 for a more thorough description). These classifications were then used to calculate the number of each type of life event the child had experienced. Two items from the original CASE-P were changed for our sample to make them more age-appropriate ("New boyfriend or girlfriend" and "Broke up with a boyfriend or girlfriend" were replaced by "Friend moved away" and "A close friend changed school"). The final questionnaire included 12 behaviour-dependent (e.g. positive: "Did well in an important test or exam", negative: "Failure of a grade") and 22 independent events (e.g. positive: "Parent started a new job", negative: "Death of Family member"). The number of life events per subscale was calculated by summing up the relevant items per category, whereas an impact score was calculated by summing the impact scores of the negative and positive life events separately per child. The following subscales were included in this study: number of positive life events, number of negative life events, number of negative/dependent, negative/independent, positive/dependent, negative/independent life events, and the impact of 
the negative and positive life events. The CASE-P displays satisfactory test-retest and interrater reliability and validity (Allen \& Rapee, 2009; Allen et al., 2012).

\section{Procedure}

Macquarie University's Human Ethics Committee approved this study. After the initial screening (STSC Approach subscale), BI and BUI children were invited to take part in this longitudinal study. Mothers provided written informed consent for their family's participation in the study and visited the university for a test session five times: twice at baseline, twice at 2-year follow-up and once at 5-year follow-up. Assessments as outlined above, and additional measures not described here, were completed. The test sessions lasted for approximately two hours. Families received $\$ 50$ and a small gift for the child after completing each test session.

\section{Data preparation and statistical analyses}

All analyses including BI were conducted based on parent-reported BI, but as the agreement between parent-reported and observed BI was not perfect (74\%), analyses were also repeated using only consistently categorized children $(n=150)$. In most cases, results of both sets of analyses were similar and therefore only results based on parent-report were reported. Where differences in significance were found, these are reported. PAS/SCAS total scores and CASE-P subscale scores were positively skewed and therefore transformed to approximate normality using a square root transformation. Due to technical problems with recording equipment and missed responses in questionnaires, there was a small amount of missing data (all $<5 \%$ ). Analyses are conducted with all available data.

A multi-method approach was taken to analyse both the direct relationships between the variables and the interplay between these variables. First, we explored whether children who were classified as BI at baseline, were more likely to experience certain types of life events or higher impact of these events compared to BUI and/or children without an anxiety disorder by means of independent $t$-tests. Second, hierarchical multiple regression analyses 
were used to explore the interplay between BI, life events, and anxiety. Five-year follow-up anxiety (SCAS anxiety symptom scores, presence of an anxiety disorder, and number of anxiety disorders subsequently) was the dependent variable in the model. As the presence of an anxiety disorder is a dichotomous variable, logistic regressions were used for this outcome variable. For the number of anxiety disorders, negative binomial (NB) regressions were used, as this variable conformed to a NB distribution. Predictors were added to the model in the following order: Baseline anxiety was entered in the first step to control for initial differences in anxiety at baseline. Life events were added in the second step. BI status was entered in the third step and the interactions between BI and life events were included in the final step to assess whether the interaction between BI and life events had additional explaining value over and above the main effects of BI and life events on anxiety. All variables were centred before interactions were calculated and analyses were run. As preliminary analyses did not show significant relationships between ethnicity and anxiety, ethnicity was not included in these models.

\section{Results}

\section{Preliminary findings.}

Table 1 shows the means and standard deviations of the CASE-P scores at 2-year follow-up separately for children that were categorized as BI and BUI at baseline. Independent $t$-tests showed that children who were categorized as BI at baseline were less likely to experience positive, $t(179)=2.35, p<.05$, and more specifically, fewer positive/dependent life events, $t(179)=2.21, p<.05$, than BUI children. The impact of these positive life events was also lower in BI children than in BUI children, $t(199)=2.34, p<.05$. Preliminary analyses also showed that co-morbidity (i.e., having a clinicial, non-anxiety 
disorder diagnosis of CSR of 4 or above), was associated with increased rates of negative life events and its impact (all $t \mathrm{~s}>2.03, p \mathrm{~s}<.05)^{2}$.

\section{Multiple regression analyses}

To examine the interplay between BI, dependent and independent life events (at 2-year follow-up), interactions between BI and life events, and anxiety (at 5-year follow-up), hierarchical multiple regression analyses were run. Separate models were tested for negative, positive, and the impact of life events.

Initial analyses showed that none of the interactions between BI and life events were significant predictors of anxiety outcomes. Therefore, we decided to test a more parsimonious model excluding these interactions, in line with recommendations of various authors (e.g. Judd \& Kenny, 1981; Meyers, Gamst, \& Guarino, 2006). As can be seen in Tables 2 to 4, across most models (and as we have reported elsewhere; REFERENCE BLINDED FOR REVIEW), baseline anxiety and BI were significantly related to anxiety at 5-year follow-up.

Negative life events and the impact of negative life events. Dependent negative life events at 2-year follow-up were significantly associated with increases with all anxiety outcomes at 5-year follow-up (i.e. anxiety symptoms, anxiety diagnosis, and number of anxiety diagnoses, even after controlling for baseline anxiety). Negative independent life events were not a significant predictor of anxiety problems at follow-up. Furthermore, a higher reported impact of negative life events at 2-year follow-up was consistently linked to worse anxiety outcomes at 5-year follow-up (i.e. increased anxiety symptoms, an anxiety

\footnotetext{
${ }^{2}$ Co-morbidity was entered in Step 1 for all multiple regressions involving negative life events and its impact as an exploratory measure. Co-morbidity was not a predictive factor ( $\mathrm{ps}>.05)$ in any of the analyses and therefore, was not included in the reported multiple regressions within the main text. The majority of co-morbid diagnoses in our sample consisted of externalising disorders (i.e., conduct disorder), which may explain its lack of predictive power for an internalising disorder such as anxiety.
} 
diagnosis, and increased number of anxiety diagnoses, even after controlling for baseline anxiety).

Positive life events and the impact of positive life events. Positive independent life events were predictive of having an anxiety disorder (after controlling for baseline anxiety), with lower numbers of reported positive life events being linked to an increased chance of having an anxiety disorder. This finding was not replicated for the other anxiety outcome variables. The number of positive dependent life events or the impact of positive life events was not a significant predictor of anxiety psychopathology.

Although there was no evidence of the hypothesized moderation of the relation between BI and anxiety outcomes by positive life events (i.e. none of the interactions between BI and life events predicted anxiety at 5-year follow-up), a relation was found between BI and positive life events on the one hand and positive life events and anxiety disorder status on the other hand. This could suggest mediation is present. Therefore, we explored whether positive life events mediated the relation between $\mathrm{BI}$ and anxiety disorder status at 5-year follow-up. The hypothesized mediation model was estimated using regression analyses in combination with a bootstrapping procedure. BI was used as the independent variable, positive dependent and independent life were the mediators, and anxiety disorder status (i.e. having an anxiety disorder or not) was the dependent variable in the model. The analysis was conducted with and without including baseline anxiety disorder status as covariate. Furthermore, the regression weights and standard errors for the direct effects (i.e. between the independent and dependent variable) were estimated in the usual way, that is, by means of regression analyses. However, this was not possible for the indirect effects (i.e. the links between the independent variable and the mediators and between the mediators and the dependent variable), because these effects have to be calculated from the product of two direct effects and the distribution of this product cannot assumed to be normal (Preacher \& Hayes, 2008). This non-normality is 
particularly problematic when the sample size is moderate, as was the case in the present study (i.e. $N=202$ ). In addition, it should be mentioned that the Sobel test, which is commonly used to examine indirect effects and mediation hypotheses, also requires a normal distribution of the variables involved. Because of the relatively moderate sample size and the intention to test the effects of two mediators simultaneously the Sobel test would not be appropriate. To explore the indirect effects and to test the mediation hypotheses, we employed Preacher and Hayes' (2008) bootstrapping procedure by means of a special macro that was programmed in SPSS. This macro was used to estimate a $95 \%$ (bias corrected) confidence interval for the total and specific indirect effects of the mediators. Results of the multiple mediation analyses however showed no evidence of positive life events mediating the relation between BI and anxiety disorder status. That is, although the total indirect effect from BI through positive dependent and independent life events to anxiety disorder status at 5-year follow-up was significant (95\% CI [.003 - .53] and 95\% CI $[.005-.52]$ after controlling for baseline anxiety disorder status), none of the specific indirect effects reached significance (all 95\% CIs included 0).

\section{Discussion}

The current study assessed the longitudinal relationships between BI, life events, and anxiety in a sample of young children. To our knowledge, this is the first study on this topic to: (1) include diagnostic interviews to establish clinical anxiety disorders next to measures of anxiety symptoms; (2) examine these relationships over an extended period of time, and (3) make a distinction between different types of life events. In line with our hypotheses, more negative life events, and negative behaviour-dependent life events in particular, as well as the impact of negative life events predicted more anxiety symptoms, increased likelihood of having an anxiety disorder diagnosis, and a greater number of anxiety diagnoses three years later. Experiencing more positive behaviour-independent life events, on the other hand, 
decreased the likelihood of an anxiety disorder diagnosis, but was not linked with anxiety symptoms or the number of anxiety disorders. Differences were also found in life events between BI and BUI children. That is, BI children experienced fewer positive events, especially behaviour-dependent positive life events. The impact of positive life events was also lower in BI children than BUI children. However, it is worth noting that this result was not replicated in analyses that only included consistently categorized children. Finally, BI did not interact with life events in the prediction of anxiety problems as hypothesized. Therefore, this study does not provide evidence for the diathesis-stress, vantage sensitivity or differential susceptibility account, but seems to indicate that BI and life events function as additive risk factors in the development of anxiety problems.

The relationships found between negative life events and anxiety is consistent with previous research showing that negative life events and their impact on children's lives are associated with increased anxiety in children (Allen et al., 2008; Boer et al., 2002; Edwards et al., 2010; Eley \& Stevenson, 2000; Goodyer et al., 1990; Gothelf et al., 2004; Rapee \& Szollos, 2002; Tiet et al., 2001). The findings are also consistent with a previous crosssectional study by Allen and Rapee (2009), which showed that anxious children experienced more negative behaviour-dependent life events. This current study extends this knowledge by providing longitudinal evidence for the contribution of negative behaviour-dependent, but not negative behaviour-independent life events, and the impact of negative life events on childhood anxiety. However, the pattern of findings also appears inconsistent with evidence from another study by Allen and colleagues (Allen et al., 2008). That study showed that severe negative independent life events and chronic adversities were also associated with the onset of clinical episodes of anxiety in children. This inconsistency may be due to differences in assessment tools selected to measure life events. Allen et al. (2008) used an investigatorbased life events interview - the Psychosocial Assessment of Child Experiences (PACE) - to 
evaluate and record life events. In contrast, the current study as well as the Allen et al. (2009) paper used the CASE checklist. The PACE is considered a 'gold-standard' for evaluating life stress, given that it can provide a more in-depth assessment of the independence of life events from the child's behaviour and to assess the contextual impact of life events in addition to their subjective impact. Therefore, the PACE may have been more sensitive in detecting the impact of severe life events, given that another study also showed a significant association between severe life events and onset of childhood psychiatric disorder (Sandberg et al., 2001). Interestingly, despite moderate to substantial agreement on the number and impact of life events documented between checklist and interview method, previous research also suggests that the CASE checklist may be more sensitive to the detection of the relationship between life events and psychopathology than the PACE interview (Allen, Rapee, \& Sandberg, 2012). Future studies may need to consider the inclusion of both measures to determine the relative strengths of each measure in documenting early life stress and its association with the onset of childhood anxiety disorders.

When focusing on negative behaviour-dependent life events and their role in child anxiety, our study showed that experiencing more negative dependent life events predicted higher anxiety symptoms and increased risk of developing anxiety disorders. This is in line with models of anxiety development that consider negative life events as a risk factor for the development of anxiety problems, through the formation and reinforcement of cognitive vulnerability (e.g. Hudson \& Rapee, 2004; Rapee, 2001). However, these models do not distinguish between behaviour-dependent and independent life events. To date, it remains unclear why anxiety is only predicted by behaviour-dependent negative life events. One possibility is that anxiety and negative-dependent life events are caused by the same shared source, for example, genetics or a vulnerable temperament, negative family environment, parental modelling, or an adverse social environment. Although more research is needed to 
clarify the process by which negative-dependent life events precipitate anxiety in children, our study suggests that the temperamental trait of BI is unlikely to be the shared cause. That is, BI was not linked to negative life events in this study (i.e., no differences were found between children categorized as BI and BUI at baseline in subsequent negative life events). One possibility is that perceived responsibility for negative life events (e.g. I am being bullied because I am no good) produces negative mood or emotion and significant distress within the child, which in its turn puts the child at risk for developing anxiety problems. Therefore, future studies are warranted to assess the subjective behaviour-dependence or independence of life events and children's cognitions around these life events, to try to clarify how behaviour-dependent life events lead to increased childhood anxiety. Differences between BI and BUI children were found on positive life events, with BI children experiencing fewer positive, and behaviour-dependent positive life events at 2-year follow-up. The impact of these positive life events was also lower in BI children than in BUI children. This suggests that BI children seem to miss out on positive life events, especially when these events depend on their behaviour (e.g. making a new friend). This is in line with research on anxious children, which showed that anxious children experience fewer behaviour-dependent positive life events (Allen \& Rapee, 2009). This strongly suggests a vicious cycle in which the fearfulness and apprehension experienced by BI children in novel situations makes them more likely to avoid these positive situations, thus causing them to miss out on various positive life experiences. The lack of positive life events in its turn could increase the vulnerability of BI children to anxiety problems by limiting opportunities to develop and practice their social and coping skills (Lyneham \& Rapee, 2004). However, in our sample, neither independent nor dependent positive life events mediated the relationship between BI and anxiety diagnosis status, which limits the contribution of positive life events to the onset of anxiety disorders for BI children. However, positive life events may act as a protective 
factor in the development of child anxiety. Results of this study seem to support this notion. That is, when children experienced more positive behaviour-independent life events, this decreased their risk of being diagnosed with an anxiety disorder. This suggests that theoretical models on childhood anxiety should not only include negative behaviourdependent life events, but also need to consider the role of positive life events in the development of anxiety.

Finally, our hypothesis that BI and life events would interact in a way consistent with the diathesis-stress, vantage sensitivity or differential susceptibility models was not supported. No interaction was found between BI and life events in the prediction of anxiety. However, BI and life events did act as independent predictors of the development of anxiety. Although inconsistent with the results of Brozina and Abela (2006) who found support for a diathesis stress account, these results are consistent with findings by Edwards (2010) and Muris and colleagues (2011), who also only found evidence for BI and life events acting as independent predictors of anxiety problems in children. Taken together, it seems that BI, as well as higher numbers of negative (behaviour-dependent) life events and higher negative impact of negative life events, and a lack of positive (behaviour-independent) life events put children at risk for the development of anxiety problems in late childhood. Future research should examine whether specific events and stressors (e.g. social versus non-social) are most important in the development of these anxiety problems.

It is also worth noting that in this study we assessed stressful life events over a 12 month period, when the children were approximately 6 years of age. Our results may differ when negative life events are assessed during different periods of development. For instance, while negative life events do not interact with temperament during childhood to predict anxiety, this may not be true for BI individuals who encounter negative life events during adolescence. Negative life events in adolescence may be quite different to events encountered 
in childhood given that adolescents are more able to influence their environment, and thus contribute more actively to the occurrence of dependent life events. However, adolescents may experience increase risk for negative life events given that they will have greater exposure to deleterious factors such as alcohol and drug use. Therefore, adolescents may be subject to more independent (e.g. peers and friends affected by drug-related accidents) as well as dependent negative life events (e.g. taking up drugs and alcohol). Future studies examining this issue in other age groups will provide important clues on the qualitative and relative contribution of negative life events, and their relationship to temperament in predicting anxiety and mood disorders across development.

Several limitations of this study need to be considered when interpreting these findings. First, only extreme groups (i.e. BI and BUI) were used, which means that the findings for this study may not be generalisable to the general population. However, when looking at anxiety and its disorders, it seems probable that these extreme groups are the most likely to develop these problems. Examining extreme groups (i.e. children scoring one SD above and below the normative mean) is further supported by Moehler et al., (2008), who have suggested that BI and BUI children belong to distinctive and discrete categories produced by different biological factors, based on the rationale that different phenotypes are often the result of distinct genotypes (see Mervielde \& De Pauw, 2012). Therefore, the long-term psychological outcomes for BI and BUI groups are important and informative.

It must be noted that results described were based in parent-report of BI, and although the good agreement between parent and observer when rating BI, some of our significant results became non-significant after rerunning the analyses with only the consistently categorized BI and BUI children. One potential explanation for this change is the decreased power to detect significant differences due to the decreased sizes for the consistently categorized groups. For instance, while negative dependent life events predicted anxiety 
diagnoses when we included all children, this effect became non-significant when we included only consistently classified children. However, our power was reduced by $18 \%$ for this predictor (negative dependent life stress) when the data was re-analysed with a smaller sample size (Hsieh, Block, \& Larsen, 1998). This is an example of a substantial drop in power and may explain the non-significant findings reported in our study with the reduced sample size. Another explanation is that parents who over- or under-report their child's BI may also show a bias in a similar direction for life events. However, it is most likely that this would affect the reported impact rating more than it would impact the report of whether specific events did or did not happen and thus not affect the number of positive or negative life events reported. As the alternative analyses mainly show differences in significance level on the number of life events, and even on behaviour-independent life events (e.g. which include items such as parents getting a divorce, pet dying, getting sick, lost or injured, or person in the family being really sick or injured), it seems more likely that another factor, such as decreased power, caused these differences.

Second, this study relied on subjective parent-report of life events, their impact and valence. Thus biases caused by parent's current mood or stress levels may have played a role(e.g. Cohen, Towbes, \& Flocco, 1988). Some researchers therefore suggests the concurrent use of interviews (e.g. Monroe \& Roberts, 1990) and objective raters to remedy such biases (i.e., positive or negative; Williamson, Birmaher, Dahl, \& Ryan, 2005). However, although future studies could benefit from a multiple methods and informants approach, other studies have shown moderate to substantial agreement on the number of life events and impact between checklists and interview measures of positive and negative life events (Allen, Rapee, \& Sandberg, 2012; Wagner, Abela, \& Brozina, 2006). Moreover, it seems that neither depressive nor anxiety symptoms led to over-reporting of events on checklists compared to interview measures (Wagner et al., 2006). Additionally, it must be kept in mind 
that, although the distinction between behaviour-dependent and -independent events in this study has been made based on previous research (Allen \& Rapee, 2009), it is possible that if more information about the specific life events in a family were made available, some of these events may have been categorized differently. Additionally, there is a lack of clarity regarding whether these behaviour-dependent events were indeed driven by anxious behaviours or behaviours unrelated to anxiety, which future studies will need to address.

Finally, we recognise that our sample has several characteristics that may not make them representative of $\mathrm{BI}$ and BUI children from the general population. First, there is considerable evidence that poverty confers a wide-array of physical and socio-emotional stress on the child, which can alter their developmental trajectory, including elevated vulnerability to psychopathology (Evans \& Kim, 2013). The majority of children in our sample, however, were from two-parent, middle-income, and educated families. Therefore, we are lacking important information from disadvantaged children in our sample. We recommend that future studies examine the relationship between BI and negative life events in socioeconomically disadvantaged groups of children.

Second, there was a differential rate of drop-outs between BI and BUI children, with greater attrition in the former group. This could have led to some bias in our results as these children and families may have possessed specific characteristics that made them more likely to drop-out of the study. For instance, these children and families may have experienced more severe anxiety or life adversities combined with BI that impeded attendance to the research sessions. This limits the generalisability of our results. Moreover, it could have impacted on the power to detect true interaction effects between BI and life events. Future studies may need to consider focusing on the retention of BI families as an important priority for longitudinal research in this area. 
To conclude, this study shows that BI and life events act as additive risk factors in the development of anxiety problems. It specifically showed that an increased number of negative behaviour dependent life events and the impact of negative life events can increase the risk of developing anxiety symptoms and anxiety disorders. Additionally, this study demonstrated a protective role for positive behaviour-independent life events in the development of child anxiety. These findings may have implications for clinical practice. For instance, within cognitive behaviour therapy, clinicians often discuss how events trigger thoughts, which then lead to feelings of anxiety in children, and associated unhelpful behaviours (i.e., avoidance). However, the events described are often articulated as independent of the child, and thus, it is unsurprising that within a clinical setting, children and families often report feeling a lack of control over stressful events. One possible solution, based on the current findings, is to increase awareness that the child's behaviour can drive future negative events. For example, a child that continues to turn down party invitations because of excessive shyness will eventually be left off the invitation list and feel excluded from friendship circles (i.e., negative dependent life events). Helping families and children to understand this cycle may allow them to reduce negative dependent life events, and increase their motivation to engage in therapeutic techniques that break this cycle. In summary, the current study elucidates an important role for life events and BI in predicting childhood anxiety disorder, and has the potential to inform frameworks for interventions that could interrupt the chronic course of anxiety disorders early in life. 


\section{References}

Allen, J. L., \& Rapee, R. M. (2009). Are reported differences in life events for anxious children and controls due to comorbid disorders? Journal of Anxiety Disorders, 23(4), $511-518$

Allen, J. L., Rapee, R. M., \& Sandberg, S. (2008). Severe life events and chronic adversities as antecedents to anxiety in children: A matched control study. Journal of Abnormal Child Psychology, 36(7), 1047-1056.

Allen, J. L., Rapee, R. M., \& Sandberg, S. (2012). Assessment of Maternally Reported Life Events in Children and Adolescents: A Comparison of Interview and Checklist Methods. Journal of Psychopathology and Behavioral Assessment, 34(2), 204-215.

Belsky, J., Bakermans-Kranenburg, M. J., \& van IJzendoorn, M. H. (2007). For better and for worse: Differential susceptibility to environmental influences. Current Directions In Psychological Science, 16, 300-304.

Belsky, J., \& Pluess, M. (2009). Beyond diathesis stress: Differential susceptibility to environmental influences. Psychological Bulletin, 135, 885-908.

Biederman, J., Rosenbaum, J. F., Bolduc-Murphy, E. A., Faraone, S. V., Chaloff, J., Hirshfeld, D. R. (1993). A 3-year follow-up of children with and without behavioral inhibition. Journal of the American Academy of Child and Adolescent Psychiatry, 32, 814-821.

Biederman, J., Rosenbaum, J. F., Chaloff, J., \& Kagan, J. (1995). Behavioural inhibition as a risk factor for anxiety disorders. In J. S. March (Ed.), Anxiety disorders in children and adolescents (pp. 61-81). New York: Guilford Press.

Boer, F., Markus, M., Maingay, R., Lindhout, I., Borst, S., \& Hoogendijk, T. (2002). Negative life events of anxiety disordered children: Bad fortune, vulnerability, or reporter bias? Child Psychiatry \& Human Development, 32(3), 187-199. 
Bradley, R. H., \& Corwyn, R. F. (2008). Infant temperament, parenting, and externalizing behavior in first grade: a test of the differential susceptibility hypothesis. Journal of Child Psychology and Psychiatry, 49, 124-131.

Broeren, S., \& Muris, P. (2008). Psychomtric evaluation of two new parent-rating scales for measuring anxiety symptoms in young Dutch children. Journal of Anxiety Disorders, $22,949-958$.

Brozina, K., \& Abela, J. R. Z. (2006). Behavioural inhibition, anxious symptoms, and depressive symptoms: A short-term prospective examination of a diathesis-stress model. Behaviour Research and Therapy, 44(9), 1337-1346.

Cohen, L. H., Towbes, L. C., \& Flocco, R. (1988). Effects of induced mood on self-reported life events and perceived and received social support. Journal of Personality and Social Psychology, 55, 669-674.

Edwards, S. L., Rapee, R. M., \& Kennedy, S. (2010). Prediction of anxiety symptoms in preschool-aged children: examination of maternal and paternal perspectives. Journal of Child Psychology and Psychiatry, 51(3), 313-321.

Eley, T. C., \& Stevenson, J. (2000). Specific life events and chronic experiences differentially associated with depression and anxiety in young twins. Journal of Abnormal Child Psychology, 28(4), 383-394.

Evans, G.W. \& Kim, P. (2013). Childhood poverty, chronic stress, self-regulation, and coping. Child Development Perspectives, 7, $43-48$.

Garnefski, N., Kraaij, V., \& Spinhoven, P. (2001). Negative life events, cognitive emotion regulation and emotional problems. Personality and Individual Differences, 30, 13111327. 
Goodyer, I., Wright, C., \& Altham, P. (1990). The friendships and recent life events of anxious and depressed school age children. British Journal of Psychiatry, 156, 689698.

Gothelf, D., Aharonovsky, O., Horesh, N., Carty, T., \& Apter, A. (2004). Life events and personality factors in children and adolescents with obsessive-compulsive disorder and other anxiety disorders. Comprehensive Psychiatry, 45(3), 192-198.

Hirshfeld-Becker, D. R., Biederman, J., \& Rosenbaum, J. F. (2004). Behavioral inhibition. In T. L. Morris \& J. S. March (Eds.), Anxiety disorders in children and adolescents (Vol. 2, pp. 27-58). New York: Guilford Press.

Hirshfeld, D. R., Rosenbaum, J. F., Biederman, J., Bolduc, E., Faraone, S. V., Snidman, N. (1992). Stable behavioural inhibition and its association with anxiety disorders. Journal of the American Academy of Child \& Adolescent Psychiatry, 31, 103-111.

Hudson, J. L., Dodd, H. F., Lyneham, H. J., \& Bovopoulous, N. (2011). Temperament and family environment in the development of anxiety disorder: Two-year follow-up. Journal of the American Academy of Child \& Adolescent Psychiatry, 50(12), 12551264.e1251.

Hsieh, F.Y., Block, D.A., \& Larsen, M.D. (1998). A simple method of sample size calculation for linear and logistic regression. Statistics in Medicine, 17, 1623-1634.

Hudson, J. L., \& Rapee, R. M. (2004). From anxious temperament to disorder: An etiological model of generalized anxiety disorder. In R. G. Heimberg, C. L. Turk \& D. S. Mennin (Eds.), The etiology and development of generalized anxiety disorder (pp. 51-74). New York: Guilford Publications.

Judd, C. M., \& Kenny, D. A. (1981). The regression discontinuity design Estimating the effects of social interventions (pp. 81-101). Cambridge: Cambridge University Press. 
Kagan, J., Reznick, J. S., Clarke, C., Snidman, N., \& Garcia-Coll, C. (1984). Behavioral inhibition to the unfamiliar. Child Development, 55, 2212-2225.

Kagan, J., Reznick, J. S., \& Gibbons, J. (1989). Inhibited and uninhibited types of children. Child Development, 60, 838-845.

Kerr, M., Lambert, W. W., Stattin, H., \& Klackenberg-Larsson, I. (1994). Stability of inhibition in a Swedish longitudinal sample. Child Development, 65, 138-146.

Lengua, L. J. (2008). Anxiousness, frustration, and effortful control as moderators of the relation between parenting and adjustment in middle-childhood. Social Development, $17,554-577$.

Lyneham, H. J., \& Rapee, R. M. (2004). Generalized anxiety disorder in children and adolescents. In S. Schneider (Ed.), Anxiety disorders in children and adolescents (pp. 197-236). Heidelberg, Germany: Springer-Verlag.

Mervielde, I. \& De Pauw, S. S. W. (2012). Models of child temperament. In M. Zentner \& R. L. Shiner (Eds.), Handbook of Temperament (pp. 21-40). NY: The Guilford Press.

Meyers, L. S., Gamst, G., \& Guarino, A. J. (2006). Multiple regression Applied multivariate research: Design and interpretation (pp. 147-196). Thousand Oaks, California: Sage Publications.

Moehler, E., Kagan, J., Oelkers-Ax, R., Brunner, R., Poustka, L., Haffner, J., \& Resch, F. (2008). Infant predictors of behavioural inhibition. British Journal of Developmental Psychology, 26, 145-150.

Monroe, S. M., \& Roberts, J. E. (1990). Conceptualizing and measuring life stress: Problems, principles, procedures, progress. Stress Medicine, 6, 209-216.

Muris, P., Merckelbach, H., Schmidt, H., Gadet, B., \& Bogie, N. (2001). Anxiety and depression as correlates of self-reported behavioural inhibition in normal adolescents. Behaviour Research and Therapy, 39, 1051-1061. 
Muris, P., Van Brakel, A. M. L., Arntz, A., \& Schouten, E. i. p. (2011). Behavioral inhibition as a risk factor for the development of childhood anxiety disorders: A longitudinal study. Journal of Child and Family Studies, 20, 157-170.

Nauta, M. H., Scholing, A., Rapee, R. M., Abbott, M., Spence, S. H., \& Waters, A. (2004). A parent report measure of children's anxiety. Behaviour Research and Therapy, 42, 813-839.

Pluess, M., \& Belsky, J. (2010). Differential susceptibility to parenting and quality child care. Developmental Psychology, 46, 379-390.

Pluess, M., \& Belsky, J. (2012). Vantage sensitivity: Individual differences in response to positive experiences. Psychological Bulletin, First Online Publication. Doi: 10.1037/a0030196.

Preacher, K. J., \& Hayes, A. F. (2008). Asymptotic and resampling strategies for assessing and comparing indirect effects in multiple mediator models. Behavior Research Methods, 40, 879-891.

Prior, M., Smart, D., Sanson, A., \& Oberklaid, F. (2000). Does shy-inhibited temperament in childhood lead to anxiety problems in adolescence. Journal of the American Academy of Child \& Adolescent Psychiatry, 30, 461-468.

Rapee, R. M. (2001). The development of generalized anxiety. In M. W. Vasey \& M. R. Dadds (Eds.), The developmental psychoptahology of anxiety (pp. 481-503). Oxford: Oxford University Press.

Rapee, R. M., Kennedy, S., Ingram, M., Edwards, S., \& Sweeney, L. (2005). Prevention and early intervention of anxiety disorders in inhibited preschool children. Journal of Consulting and Clinical Psychology, 73, 488-497.

Rapee, R. M., \& Szollos, A. A. (2002). Developmental antecedents of clinical anxiety in childhood. Behaviour Change, 19(03), 146-157. 
Reznick, J. S., Gibbons, J., Johnson, M. O., \& McDonough, P. M. (1989). Behavioural inhibition in a normative sample. In J. S. Reznick (Ed.), Perspectives on behavioural inhibition (pp. 25-49). Chicago: University of Chicago Press.

Robinson, J. L., Kagan, J., Reznick, J. S., \& Corley, R. (1992). The heritability of inhibited and uninhibited behavior: A twin study. Developmental Psychology, 28, 1030-1037.

Sandberg, S., McGuinness, D., Hillary, C., \& Rutter, M. (1998). Independence of childhood life events and chronic adversities: a comparison of two patient groups and controls. Journal of the American Academy of Child and Adolescent Psychiatry, 37, 728-735.

Sandberg, S., Rutter, M., Pickles, A., McGuinness, D., \& Angold, A. (2001). Do high-threat life events really provoke the onset of psychiatric disorder in children? Journal of Child Psychology and Psychiatry and Allied Disciplines, 42, 523-532.

Sanson, A., Smart, D., Prior, M., Oberklaid, F., \& Pedlow, R. (1994). The structure of temperament form age 3 to 7 years: Age, sex, and sociodemographic influences. Merrill-Palmer Quarterly, 40, 233-252.

Scarpa, A., Raine, A., Venables, P. H., \& Mednick, S. A. (1995). The stability of inhibited/uninhibited temperament from ages 3 to 11 years in Mauritian children. Journal of Abnormal Child Psychology, 23, 607-619.

Shahar, G., \& Priel, B. (2002). Positive life events and adolescent emotional distress: in search of protective-interactive processes. Journal of Social and Clinical Psychology, $21,645-668$.

Silverman, W. K., \& Nelles, W. B. (1988). The Anxiety Disorders Interview Schedule for Children. Journal of the American Academy of Child and Adolescent Psychiatry, 27, $772-778$.

Spence, S. H., Rapee, R. M., McDonald, C., \& Ingram, M. (2001). The structure of anxiety symptoms among preschoolers. Behaviour Research \& Therapy, 39, 1293-1316. 
Stright, A. D., Gallagher, K. C., \& Kelley, K. (2008). Infant temperament moderates relations between maternal parenting in early childhood and children's adjustment in first grade. Child Development, 79, 186-200.

Tiet, Q. Q., Bird, H. R., Hoven, C. W., Moore, R., Wu, P., Wicks, J. (2001). Relationship between specific adverse life events and psychiatric disorders. Journal of Abnormal Child Psychology, 29(2), 153-164.

Turner, S. M., Beidel, D. C., \& Wolff, P. L. (1996). Is behavioural inhibition related to anxiety disorders? Clinical Psychology Review, 16, 157-172.

van Aken, C., Junger, M., Verhoeven, M., van Aken, M. A. G., \& Deković, M. (2007). The interactive effects of temperament and maternal parenting on toddlers' externalizing behaviours. Infant and Child Development, 16, 553-572.

Wagner, C., Abela, J., \& Brozina, K. (2006). A Comparison of Stress Measures in Children and Adolescents: A Self-Report Checklist Versus an Objectively Rated Interview. Journal of Psychopathology and Behavioral Assessment, 28(4), 250-260.

Williamson, D. E., Birmaher, B., Dahl, R. E., \& Ryan, N. D. (2005). Stressful life events in anxious and depressed children. Journal of Child and Adolescent Psychopharmacology, 15(4), 571-580. 
Table 1

Prevalence rates and comparison of BI and BUI groups on number of anxiety diagnoses, anxiety scores and life events at baseline and follow-up

\begin{tabular}{|c|c|c|c|c|}
\hline & \multicolumn{2}{|c|}{ Baseline } & \multicolumn{2}{|c|}{ 5-year follow-up } \\
\hline & BI & BUI & $\mathrm{BI}$ & BUI \\
\hline & $(n=102)$ & $(n=100)$ & $(n=71)$ & $(n=89)$ \\
\hline Any anxiety disorder & $73 \%$ & $17 \%$ & $\overline{54 \%}$ & $18 \%$ \\
\hline Social Phobia & $43 \%$ & $0 \%$ & $37 \%$ & $3 \%$ \\
\hline Separation Anxiety Disorder & $30 \%$ & $2 \%$ & $10 \%$ & $1 \%$ \\
\hline Specific Phobia & $50 \%$ & $12 \%$ & $21 \%$ & $11 \%$ \\
\hline Generalised Anxiety Disorder & $12 \%$ & $3 \%$ & $21 \%$ & $9 \%$ \\
\hline Obsessive Compulsive Disorder & $2 \%$ & $2 \%$ & $0 \%$ & $0 \%$ \\
\hline Post Traumatic Stress Disorder & $2 \%$ & $0 \%$ & $0 \%$ & $0 \%$ \\
\hline Mean number anxiety diagnoses & $1.59(1.38)$ & $0.24(0.55)$ & $1.04(1.26)$ & $0.25(0.67)$ \\
\hline \multirow[t]{4}{*}{ Mean PAS scores } & $35.99(16.02)$ & $11.36(9.40)$ & $20.77(12.13)$ & $11.84(7.46)$ \\
\hline & \multicolumn{2}{|c|}{ 2-year follow-up } & & \\
\hline & $\mathrm{BI}$ & BUI & & \\
\hline & $(n=87)$ & $(n=91)$ & & \\
\hline Life events & $M(S D)$ & $M(S D)$ & & \\
\hline Positive & $2.42(0.45)^{*} \dagger$ & $2.59(0.58)^{*} \dagger$ & & \\
\hline Negative & $1.14(0.90)$ & $1.29(0.94)$ & & \\
\hline Positive/dependent & $1.51(0.46)^{*} \dagger$ & $1.65(0.41)^{*} \dagger$ & & \\
\hline Positive/independent & $1.37(0.55)$ & $1.50(0.52)$ & & \\
\hline Negative/dependent & $0.36(0.53)$ & $0.35(0.62)$ & & \\
\hline
\end{tabular}


RUNNING HEAD: TEMPERAMENT, STRESSFUL LIFE EVENTS, AND ANXIETY

Negative/independent

$1.02(0.84) \quad 1.94(0.80)$

Impact negative events

$12.28(10.82) \quad 11.34(12.76)$

Impact positive events

$7.75(4.95)^{*}+$

$9.30(4.48)^{*} \dagger$

Note. PAS $=$ Preschool Anxiety Scale. ${ }^{*} p<.05 . \dagger=$ non-significant when analyses were run with only consistently categorized BI children. 
Table 2

Final multiple hierarchical regression models for BI, life events and anxiety scores

\begin{tabular}{|c|c|c|c|c|}
\hline Variables & $\Delta R^{2}$ & $\Delta F$ & $\beta$ & $t$ \\
\hline \multicolumn{5}{|l|}{ Anxiety scores: Negative life events } \\
\hline Step 1 & $.39 * * *$ & 92.53 & & \\
\hline Baseline anxiety scores & & & .63 & $9.62 * * *$ \\
\hline Step 2 & $.04 * *$ & 4.96 & & \\
\hline Baseline anxiety scores & & & .57 & $8.73 * * *$ \\
\hline Negative dependent life events & & & .14 & $2.09 *$ \\
\hline Negative independent life events & & & .11 & 1.60 \\
\hline Step 3 & .00 & .00 & & \\
\hline Baseline anxiety scores & & & .57 & $6.04 * * *$ \\
\hline Negative dependent life events & & & .14 & $2.07 *$ \\
\hline Negative independent life events & & & .11 & 1.59 \\
\hline Behavioural Inhibition & & & .00 & 0.02 \\
\hline Total $R^{2}$ & $.43 * * *$ & & & \\
\hline
\end{tabular}

Anxiety scores: Positive life events

Step 1

Baseline anxiety scores

Step 2

Baseline anxiety scores

Positive dependent life events

Positive independent life events

Step 3

Baseline anxiety scores

Positive dependent life events

$.39 * * * \quad 92.53$

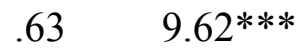

.00

.26

$\begin{array}{ll}.62 & 9.37 * * * \\ -.04 & -.55 \\ -.02 & -.34\end{array}$

$.00 \quad .36$ 
Positive independent life events

$-.03 \quad-.37$

Behavioural Inhibition

$-.06-.60$

Total $R^{2}$

$.40 * * *$

Anxiety scores: Impact negative life events

Step 1

$.39 * * * \quad 92.53$

Baseline anxiety scores

$.639 .62 * * *$

Step 2

$.03 * * \quad 6.65$

Baseline anxiety scores

$.598 .97 * * *$

Impact negative life events

$.17 \quad 2.58^{* *}$

Step 3

.00

.03

Baseline anxiety scores

$.60 \quad 6.38 * * *$

Impact negative life events

$.17 \quad 2.52 * *$

Behavioural Inhibition

$\begin{array}{ll}-.02 & -.17\end{array}$

Total $R^{2}$

$.42 * * *$

Anxiety scores: Impact positive life events

Step 1

$.39 * * * \quad 91.09$

Baseline anxiety scores

.62

$9.54 * * *$

Step 2

.00

.95

Baseline anxiety scores

$629.50 * * *$

Impact positive life events

$.06 \quad 0.98$

Step 3

.00

.18

Baseline anxiety scores

$65 \quad 6.90 * * *$

Impact positive life events

$.06 \quad 0.91$

Behavioural Inhibition

$-.04 \quad-.42$

Total $R^{2}$ $.39 * * *$

Note. Behavioural inhibition is coded $0=$ Uninhibited, $1=$ Inhibited.

$* * * p<.001, * * p<.01, * p<.05$ 
Table 3

Final multiple hierarchical logistic regression models for BI, life events and presence of an anxiety disorder

\begin{tabular}{|c|c|c|c|c|c|}
\hline Variables & $\chi^{2}$ block & $b$ & $S E$ & Wald & OR \\
\hline \multicolumn{6}{|l|}{ Anxiety disorder: Negative life events } \\
\hline Step 1 & $16.39 * * *$ & & & & \\
\hline Baseline anxiety scores & & -1.42 & .36 & $15.63 * * *$ & .24 \\
\hline Step 2 & $7.43 *$ & & & & \\
\hline Baseline anxiety scores & & -1.39 & .37 & $13.98 * * *$ & 0.25 \\
\hline Negative dependent life events & & .83 & .33 & $6.47 * * \dagger$ & 2.29 \\
\hline Negative independent life events & & .00 & .25 & .05 & 1.00 \\
\hline Step 3 & $12.51 * * *$ & & & & \\
\hline Baseline anxiety scores & & -.71 & .43 & 2.77 & .49 \\
\hline Negative dependent life events & & .94 & .34 & $7.79 * * * \dagger \dagger$ & 2.56 \\
\hline Negative independent life events & & .03 & .25 & 0.02 & 1.03 \\
\hline Behavioural Inhibition & & -1.51 & .44 & $11.87 * * *$ & .22 \\
\hline Total chi-square & $36.33 * * *$ & & & & \\
\hline
\end{tabular}

Anxiety disorder: Positive life events

Step 1

Baseline anxiety scores

Step 2

Baseline anxiety scores

Positive dependent life events

Positive independent life events

Step 3

Baseline anxiety scores
$16.39 * * *$

$$
\begin{array}{llll}
-1.42 & .36 & 15.63 * * * & .24
\end{array}
$$

$9.54 * *$

$\begin{array}{cccc}-1.40 & .37 & 14.22 * * * & .25 \\ -.60 & .40 & 2.27 & .55 \\ -.78 & .35 & 4.97 * & .46\end{array}$

$8.24 * *$

$\begin{array}{lll}-.87 \quad .42 & 4.26^{* \dagger}+.42\end{array}$


Positive dependent life events

Positive independent life events

Behavioural Inhibition

Total chi-square

$\begin{array}{llll}-.48 & .42 & 4.26 * \dagger & .42 \\ -.72 & .36 & 4.06 * \dagger \dagger & .49 \\ -1.21 & .43 & 8.10 * * & .30\end{array}$

$34.17 * * *$

Anxiety disorder: Impact negative life events

Step 1

Baseline anxiety scores

Step 2

Baseline anxiety scores

Impact negative life events

Step 3

Baseline anxiety scores

Impact negative life events

Behavioural Inhibition

Total chi-square
$16.39 * * *$

$\begin{array}{llll}-1.42 \quad .36 & 15.63 * * * & .24\end{array}$

$4.97 *$

$$
\begin{array}{cccc}
-1.35 & .37 & 13.62 * * * & .26 \\
.21 & .10 & 4.75 & 1.24
\end{array}
$$

$11.75^{* * *}$

$\begin{array}{cccc}-.69 & .42 & 2.69 & .50 \\ .24 & .10 & 5.61 * & 1.27 \\ -1.44 & .43 & 11.30 * * * & .24\end{array}$

$33.12 * * *$

Anxiety disorder: Impact positive life events

Step 1

Baseline anxiety scores

Step 2

Baseline anxiety scores

Impact positive life events

Step 3

Baseline anxiety scores

Impact positive life events

Behavioural Inhibition
$17.869 * * *$

$\begin{array}{llll}-1.46 & .36 & 16.93 * * * & .23\end{array}$

$\begin{array}{cccc}-1.45 & .36 & 16.48 * * * & .23 \\ -.32 & .21 & 2.35 & .73\end{array}$

$9.53 * *$

$\begin{array}{cccc}-.90 & .40 & 4.90^{*} & .41 \\ -.32 & .22 & 2.20 & .73 \\ -1.25 & .41 & 9.36 * * & .29\end{array}$

Total chi-square $29.80 * * *$

Note. Behavioural inhibition is coded $0=$ Uninhibited, $1=$ Inhibited. 
$* * * p<.001, * * p<.01, * p<.05 . \dagger=$ non-significant when analyses are run with only consistently categorized BI children, $\dagger \dagger=$ borderline significant when analyses are run with only consistently categorized BI children. 
Table 4

Final multiple negative binomial regression models for BI, life events and number of anxiety disorders

$\begin{array}{lllll}\text { Variables } & b & S E & \text { Wald } & \text { IRR }\end{array}$

Number of anxiety disorders: Negative life events

Baseline anxiety scores

Negative dependent life events

Negative independent life events

Behavioural Inhibition

Likelihood ratio chi-square

$\begin{array}{rccc}.29 & .13 & 5.33 * \dagger & 1.34 \\ .54 & .25 & 4.56 * \dagger & 1.71 \\ .09 & .18 & .26 & 1.10 \\ -1.23 & .34 & 12.71 * * * & .29\end{array}$

$41.36^{* * *}$

Number of anxiety disorders: Positive life events

Baseline anxiety scores

Positive dependent life events

Positive independent life events

Behavioural Inhibition

Likelihood ratio chi-square

$\begin{array}{llll}.34 & .13 & 7.43 * * \dagger & 1.41\end{array}$

$\begin{array}{llll}-.21 & .28 & .57 & .81\end{array}$

$\begin{array}{llll}-.26 & .25 & 1.03 & .78\end{array}$

$-1.00 \quad .34 \quad 8.85 * *$

Number of anxiety disorders: Impact negative life events

$\begin{array}{lcccc}\text { Baseline anxiety scores } & .28 & .13 & 4.97 * \dagger & 1.32 \\ \text { Impact negative life events } & .19 & .08 & 6.03 * & 1.21 \\ \text { Behavioural Inhibition } & -1.20 & .34 & 12.33 * * * & .30\end{array}$

Likelihood ratio chi-square

$41.66 * * *$

Number of anxiety disorders: Impact positive life events

Baseline anxiety scores

Impact positive life events

Behavioural Inhibition

Likelihood ratio chi-square

$\begin{array}{cccc}.34 & .12 & 7.78 * * & 1.41 \\ -.08 & .16 & .22 & .92 \\ -.98 & .32 & 9.07 * * & .38\end{array}$

$34.09 * * *$

Note. Behavioural inhibition is coded $0=$ Uninhibited, $1=$ Inhibited. 
$* * * p<.001, * * p<.01, * p<.05 . \dagger=$ non-significant when analyses are run with only consistently categorized BI children. 